\title{
The Benefit of Archival Research in Investigating Dwarf Novae
}

\author{
C. la Dous
}

ESA/IUE Observatory, Villafranca del Castillo, Apartado 50727, 28080 Madrid, Spain

\section{Introduction}

Dwarf novae and nova-like stars are a sub-group of the class of cataclysmic variables. Most of these stars show high and low brightness states (maxima or outbursts and minima or quiescent states, respectively). They all are short-period interacting binaries consisting of a white dwarf primary, a late-type Roche-lobe filling secondary, and an accretion disk around the white dwarf. For details on observations of cataclysmic variables and their theoretical explanations and modelling, the interested reader is referred to recent reviews.

The main problem when trying to understand these systems is the wealth of patterns presented by the whole group: no two objects are nearly identical and it is hard to say which the 'typical' features are. As a result of this, from an individual observation, or from observations of a few systems only, it is not possible to decide which of the observed features are characteristic of the entire class, and which are specific of individual members. The only way is to investigate many different members of the same class, arrange them according to their different physical parameters, and try to determine what the general characteristics are.

So far, data archives of the size and accessibility required for statistical investigations do not exist in the optical. Fortunately, from the very beginning of the IUE project, all observations were archived in a homogeneous way, together with the tools for a proper reduction and calibration; since many years the low-resolution UV spectra are available through ULDA, basically on-line, in a fully calibrated form. For dwarf novae and nova-like stars, more than 2000 spectra of over 100 objects have so far been accumulated (la Dous 1990). With this data set, which covers nicely the parameter space, it is possible to carry out a statistical investigation like the one discussed above. An additional help that proved to be crucial is the series of visual observations made by members of the American Association of Variable Star Observers (AAVSO) which allow a rather exact phasing of the observations in the outburst light curve. 


\section{Results}

Without going into details, in the following I will summarize the most important results of the statistical analysis of low-resolution IUE spectra of dwarf novae and non-magnetic nova-like stars. Details of these investigations are given in la Dous $(1991 ; 1994 ; 1995$, in preparation).

\subsection{The continum radiation at maximum}

For dwarf novae at maximum, and likewise for nova-like stars in the high brightness state, it is assumed that the accretion disk is stationary. Thus, assuming a value for the mass-transfer rate and for some other less important parameters, the continuum spectrum of the radiation emitted by the disk can be computed. If the continuum flux distributions of dwarf novae and nova-like stars at the peak of an outburst and in the high brightness state are compared with each other and with theoretical models, the following picture emerges: some $80 \%$ of the dwarf novae have approximately identical continuum flux distributions which are systematically redder than predicted for a stationary disk. In the case of nova-like stars, the internal agreement between the flux distributions of the different members of the class is not as good as in the case of dwarf novae, but with very few exceptions all of them are considerably redder, i.e., cooler, than expected on theoretical grounds. The reasons for this disagreement are not yet clear, but it is obvious that some mechanism is extracting energy from the continuum radiation of the accretion disk. Possible candidates are winds or magnetic loops forming above the disk, but the question remains open.

\subsection{The line radiation at maximum}

The pattern of line radiation emitted by accretion disks in the high brightness state ranges from strong emission lines to strong absorption lines with $\mathrm{P}$ Cygni profiles, to about featureless continua. The most obvious physical parameter on which a dependence is to be expected is the inclination angle of the system. If the line strengths of all observed systems are arranged according to this angle, and if we accept that one system - even if it is the brightest and best-known (SS Cyg) - does not follow the general trend, a very clear pattern emerges: low-inclination systems (where the disk is seen essentially pole-on) show strong absorptions of the UV resonance lines and strong P Cygni profiles, in particular in C IV 1550 $\AA$; as the inclination increases, the absorption lines, as well as both the emission and the absorption components of the P Cygni lines, gradually disappear. In the case of dwarf novae, around an inclination of some $60^{\circ}$ or $70^{\circ}$ a basically featureless continuum is left; only at very high inclinations, when the disk is seen edge-on, very strong and broad emissions of the resonance lines appear. In the case of non-magnetic nova-like stars the pattern is essentially identical, the only difference being that the emission components already appear when the absorptions have not yet totally disappeared. The immediate interpretation of this behaviour is that, in addition to the optically thick disk at outburst 
maximum, a wind exists which is ejected, to first approximation, perpendicularly to the disk. In addition, there must be an extended stationary corona above the disk which is responsible for the strong emission lines seen at high inclinations; this corona must be considerably larger in nova-like stars than in dwarf novae.

\subsection{The line radiation at minimum}

If we look for a similar dependence on inclination of the line radiation of dwarf novae at minimum, the emerging pattern is that at low inclinations the UV resonance lines are seen as strong emissions; as the inclination increases, the line strength gradually decreases, leaving again an essentially featureless continuum at high inclinations. The obvious interpretation is that the quiescent disk is at least partially optically thick, in particular in the hot inner areas.

\subsection{The continuum radiation at minimum}

When the continuum radiation is considered as a function of the inclination angle, it becomes obvious that at low inclinations the continuum flux consists of two components, a blue one at short wavelengths and a red one at long wavelengths; the wavelength at which the major contribution changes from one to the other varies from object to object. Furthermore, if different spectra of the same system are inspected for this feature, it results that the turn-over point moves to longer wavelengths as the overall flux level decreases. From another investigation (la Dous in preparation) it results that the temperature of the white dwarf, and whether it is of type DA or DB, can be determined with rather high accuracy from the short-wavelength $I U E$ spectrum alone. Thus, in the case of dwarf novae at minimum, supposing that the bluer one of the two continua is that of the white dwarf primary - an assumption which is supported by the presence of a strong Ly $\alpha$ absorption in several systems - the temperatures and types of these stars can be determined. If their contribution to the emitted flux is subtracted from the spectrum, what remains must be the spectrum of the accretion disk alone, which turns out to be that of an optically thick emitter. In all cases, the overal shape of the supposed disk spectrum is the same, except for the fact that the amount of the excess continuum flux decreases as the inclination angle increases, in agreement with the disk being basically a two-dimensional object which contributes progressively less to the overall flux as is seen more and more edge-on.

\section{References}

la Dous C., 1990, SSR 52, 203

la Dous C., 1991, A\&A 252, 100

la Dous C., 1994, SSR 67, 1 